\title{
Nearly integrable turbulence and rogue waves in disordered nonlinear Schrödinger systems
}

\author{
Zhi-Yuan Sun ${ }^{1,2, *}$ and Xin Yu ${ }^{1, \dagger}$ \\ ${ }^{1}$ Institute of Fluid Mechanics, Beihang University, Beijing 100191, China \\ ${ }^{2}$ International Research Institute for Multidisciplinary Science, Beihang University, Beijing 100191, China
}

(Received 29 October 2020; revised 21 April 2021; accepted 12 May 2021; published 2 June 2021)

\begin{abstract}
Integrable nonlinear Schrödinger (NLS) systems provide a platform for exploring the propagation and interaction of nonlinear waves. Extreme events such as rogue waves (RWs) are currently of particular interest. However, the presence of disorder in these systems is sometimes unavoidable, for example, in the forms of turbulent current in the ocean and random fluctuation in optical media, and its influence remains less understood. Here, we report numerical experiments of two nearly-integrable NLS equations with the effect of disorder showing that the probability of RW occurrence can be significantly increased by adding weak system noise. Linear and nonlinear spectral analyses are proposed to qualitatively explain those findings. Our results are expected to shed light on the understanding of the interplay between disorder and nonlinearity, and may motivate new experimental works in hydrodynamics, nonlinear optics, and Bose-Einstein condensates.
\end{abstract}

DOI: 10.1103/PhysRevE.103.062203

\section{INTRODUCTION}

Integrable turbulence (IT), as introduced by Zakharov [1], studying the propagation and interaction of random waves in nonlinear integrable systems, has attracted much attention from both of the theoretical and experimental communities [2-8]. It may provide an opportunity to understand sophisticated turbulent phenomena to some extent. One classical, but ubiquitous integrable model is the one-dimensional nonlinear Schrödinger equation (NLSE) which is widely used to describe nonlinear waves in deep water, optical media, Bose-Einstein condensates, and many others $[9,10]$. This equation, depending on the relative signs of its dispersion and nonlinearity, can exhibit focusing or defocusing properties that significantly influence the evolution of the IT wave field [10].

For the focusing NLSE, an important mechanism to generate IT is the noise-induced modulation instability (MI), a process transforming the plane wave, by amplifying the weak random noise superimposed on it, into a chaotic wave field characterized by a stationary single-point statistics for the long-time evolution [3,10-14]. This stationary state contains numbers of highly nonlinear localized structures, such as the Akhmediev breathers, Peregrine soliton, and Kuznetsov-Ma solitons, and the interactions among them may randomly produce high-amplitude entities that emerge with low probability, coined rogue waves (RWs) [15-17].

The term RW was initially used to label unexpected and destructive giant oceanic waves [18-20], but has now

\footnotetext{
*sunzhiyuan137@aliyun.com

†yuxin@buaa.edu.cn
}

Published by the American Physical Society under the terms of the Creative Commons Attribution 4.0 International license. Further distribution of this work must maintain attribution to the author(s) and the published article's title, journal citation, and DOI. been generalized to represent large-amplitude waves in various different systems [21-26]. It should be emphasized that the mechanisms for RW generation are in fact manyfaceted [19-22,27,28], from linear to nonlinear effects, with in particular the MI standing as an eminent example of the IT phenomena.

Nevertheless, the noise-induced MI mechanism seems to be less promising in producing a heavy-tailed probability distribution of the wave amplitudes. Instead, its stationary turbulent state has a Rayleigh distribution, such that the probability of RW occurrence is just the same as for linear waves with random phases [3]. On the other hand, the complete integrability is too specific a feature. Any nonintegrable correction to the NLSE, even in a small amount, may significantly change the fashion of RW formation. Investigations on these aspects remain active and the answers are far from satisfactory.

In the present work, we consider stochastic effects of the NLS systems on the generation of RWs. We perform numerical experiments on two prototypes of disordered NLSEs, with the weak randomness acting on the linear potential and on the cubic nonlinearity. Starting from a plane wave, the universal regimes of nonstationary turbulence are demonstrated where the probability of the occurrence of RWs is significantly increased in evolution. We give qualitative explanations to our findings. Different from the wave packet spreading in weak turbulence [29-32], presented are systematic results regarding the relatively strong turbulence and its RW content in disordered NLS systems.

\section{MODEL AND METHOD}

The model of the disordered NLSE used is written in its dimensionless form

$$
i \frac{\partial \psi}{\partial \xi}=-\frac{1}{2} \frac{\partial^{2} \psi}{\partial \tau^{2}}-g(\tau)|\psi|^{2} \psi+\varepsilon(\tau) \psi,
$$


where the complex wave field $\psi(\xi, \tau)$ is a function of the evolution variable $\xi$ and transverse variable $\tau$. We consider two typical but fundamentally different cases with the random noise playing the role of a linear and a nonlinear potential respectively,

$$
\begin{array}{ll}
\text { Case 1: } & g(\tau) \equiv 1, \quad \varepsilon(\tau)=\epsilon(\tau), \\
\text { Case 2: } & g(\tau)=1+\epsilon(\tau), \quad \varepsilon(\tau) \equiv 0,
\end{array}
$$

where the real random function $\epsilon(\tau)$ is very small, having standard deviation $\sigma \ll 1$, with zero mean and is an uncorrelated function, namely, $\langle\epsilon(\tau)\rangle=0$ and $\left\langle\epsilon(\tau) \epsilon\left(\tau^{\prime}\right)\right\rangle=$ $\sigma^{2} \delta\left(\tau-\tau^{\prime}\right)$. We used Gaussian distribution of $\epsilon(\tau)$ [we also examined the uniform distribution of $\epsilon(\tau)$ and did not find an essential difference for our results]. The initial condition was taken as a constant background of unit amplitude, $\psi(0, \tau)=1$. As a comparison, the well-studied IT is denoted as Case 0 , where $g(\tau) \equiv 1, \varepsilon(\tau) \equiv 0$, and $\psi(0, \tau)$ is composed of the constant unit amplitude with some weak noise on it.

The NLS-type equations with random effects have their own advantages in providing platforms for exploring the competition between the effects of disorder and those of nonlinearity [29-37]. In particular, Case 1 is relevant for light transport in optical media with random linear properties [38], and also for dynamics of cold atoms in disordered potentials [39,40]; Case 2 can describe the propagation of electromagnetic waves in a one-dimensional structure with random nonlinear coefficients [41,42] (see examples in Appendix A). One thus may expect results corresponding to those related areas.

The turbulent wave field was simulated by integrating Eq. (1) using the fourth-order split-step method [9] in a periodic box of the size $L \simeq 820$, and the weak random noise was chosen as the level of $\sigma=4.5 \times 10^{-3}$ (some computational details can be found in Appendix B). We implemented the simulation up to $\xi=300$ where the MI-induced IT (Case 0) has already developed to its asymptotic stationary state. In our numerical experiments, we use ensembles of 1000 realizations of $\epsilon(\tau)$ to decide the statistical quantities. We also checked these results by varying the box size, as well as by employing different integration schemes, and found no changes.

\section{RESULTS AND ANALYSES}

Figure 1 shows partial the wave field $|\psi(\xi, \tau)|$ for a specific realization of the randomness. The early stage for both cases is similar to (but not the same as) that of the IT, where one can see the emergence of quasiperiodic structures resembling the well-known Akhmediev breathers [15,16]. However, with $\xi$ increasing, the wave content will switch from breathers to mainly solitons, but in different ways for Cases 1 and 2, as will be revealed later. These coherent excitations (breathers and solitons) move and interact on the "sea of small radiation waves," as seen in Fig. 1(c), where the difference between the amplitudes of the solitons and radiation waves may come up to 1-2 orders of magnitude. We stress that this is a regime of moderately strong turbulence, with the nonlinear energy and the kinetic energy being of almost the same magnitude.
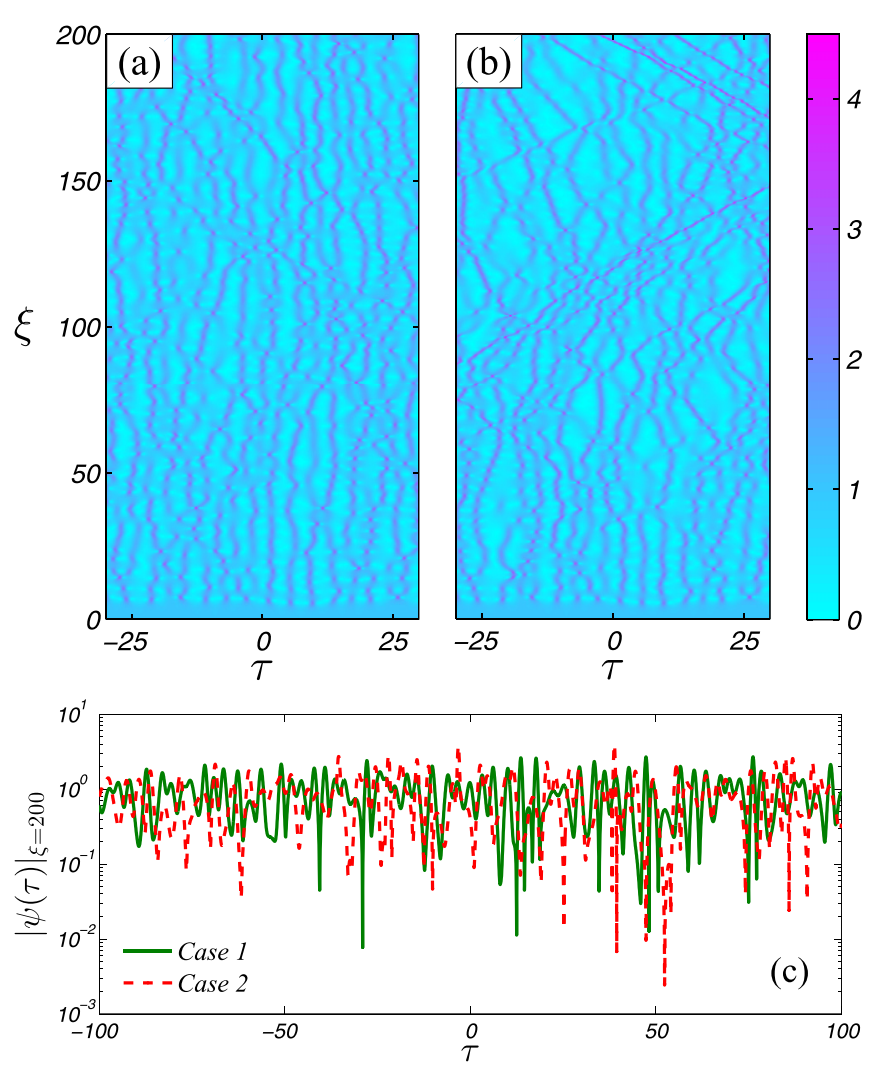

FIG. 1. Typical evolution of the turbulent wave field $|\psi(\xi, \tau)|$ for a specific realization of $\epsilon(\tau)$. (a) Case 1 with the random linear potential; (b) Case 2 with the random coefficient of the cubic nonlinearity. (c) Logarithm plots of $|\psi(\tau)|$ at $\xi=200$. The green solid line is for Case 1 and the red dashed line is for Case 2.

A statistical indicator of the turbulent wave field is the moment of the amplitude $|\psi(\xi, \tau)|$ :

$$
M_{n}(\xi)=\frac{1}{L} \int_{-L / 2}^{L / 2}\left\langle|\psi(\xi, \tau)|^{n}\right\rangle d \tau .
$$

In particular, the fourth-order moment $M_{4}$ is of interest since large values of this moment imply a heavy-tailed amplitude distribution with higher probability of extreme events [43]. Figure 2 presents the evolution of $M_{4}$ with $\xi$ for Cases 1 and 2, as compared with Case 0 . Up to $\xi \sim 20$, the forms of decaying oscillations are similar for all three cases, corresponding to

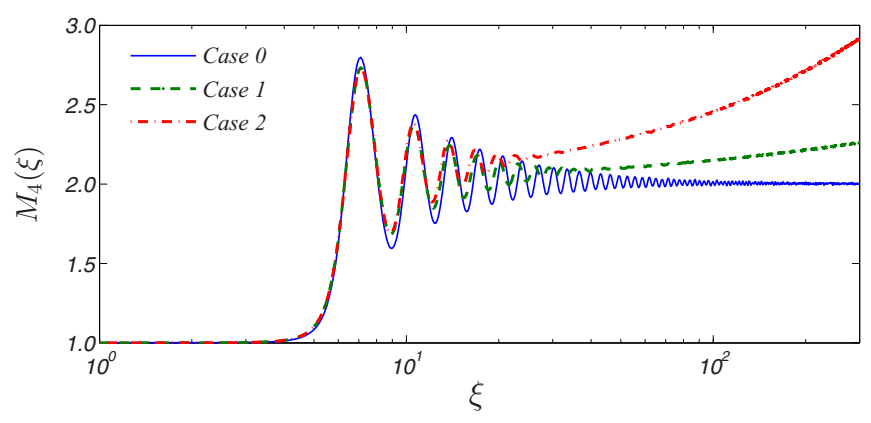

FIG. 2. Evolution of the fourth-order moment $M_{4}(\xi)$ for Case 0 (blue solid line), Case 1 (green dashed line), and Case 2 (red dotdashed line). 

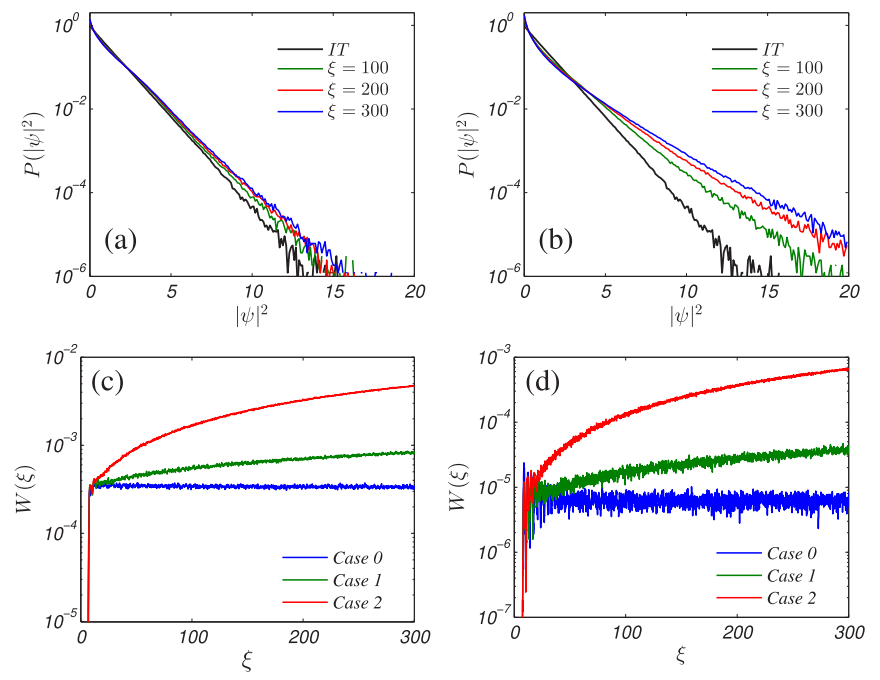

FIG. 3. Probability density functions $P\left(|\psi|^{2}\right)$ of the wave intensity $|\psi|^{2}$ at different evolution distances for (a) Case 1, (b) Case 2. The asymptotic stationary distribution for IT (Case 0 measured at $\xi=200$ ) is shown with the lowest black solid lines. Other lines, from bottom to top, correspond to $\xi=100$ to 300 . Probability of the occurrence of waves $W(\xi)$ with intensities (c) $|\psi|^{2}>8(h=8)$, (d) $|\psi|^{2}>12(h=12)$. The lines from bottom to top correspond to Cases 0 to 2 .

the dominant nonlinear stage of MI. However, for longer $\xi$, both of the disordered cases remarkably deviate from the asymptotic state of the IT, with $M_{4}$ continuously increasing. This offers us a common regime of nonstationary turbulent wave field, where the larger $M_{4}$ indicates a higher probability of RW occurrence.

We note that the computational limit $\xi=300$ units is perhaps long enough for an experimental observation. Nevertheless, the asymptotic states of Cases 1 and 2 as $\xi \rightarrow+\infty$ still seem to be an open challenge theoretically. For deterministic nonintegrable systems, the long-time formation of large-scale coherent structures on a small-scale wave turbulence was numerically observed, and this state was assumed to asymptotically reach their statistical equilibrium modes [44-47]. How these clues relate to our disordered nearly integrable systems needs on the other hand a further investigation, which is not within the scope of this work.

Figures 3(a) and 3(b) display the probability density function (PDF) of the wave intensity $|\psi(\xi, \tau)|^{2}$ measured at different evolution distances. For both Cases 1 and 2, the tails of the PDF gradually increase with $\xi$. They are apparently higher than that of the exponential distribution. In particular, at fixed $\xi$, the tail is more elevated for Case 2, which implies that the probability of RWs is more increased if the random noise is imposed on the cubic nonlinearity.

We give the probability of the occurrence of waves with their intensities exceeding a threshold $|\psi|^{2}>h$ :

$$
W(h, \xi)=\int_{h}^{+\infty} P\left(|\psi|^{2}, \xi\right) d|\psi|^{2} .
$$

For MI-induced IT, the criterion of $h \geqslant 8$ was considered as the sign of RWs [3]. Figure 3(c) presents the variation of $W(\xi)$ with $\xi$ for $h=8$. One can clearly find that the probability of
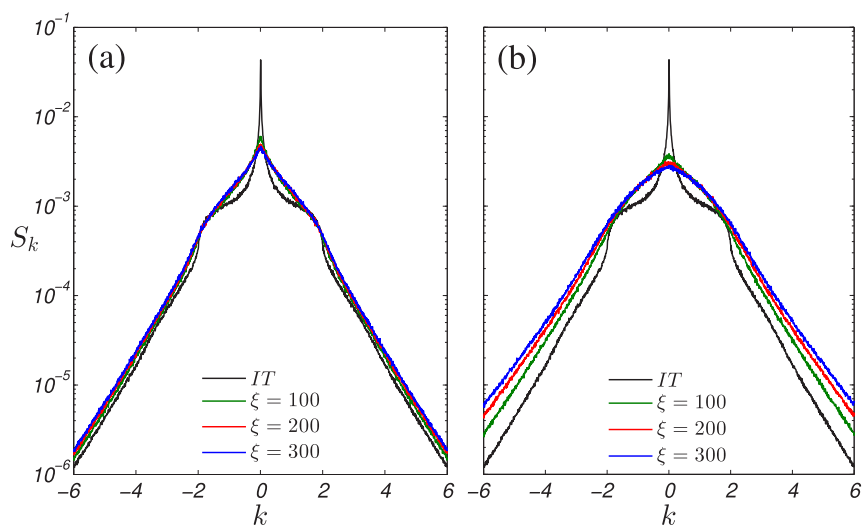

FIG. 4. The wave spectra $S_{k}$ at different evolution distances for (a) Case 1, (b) Case 2. The sharp black solid line denotes the asymptotic stationary spectrum for IT (Case 0). The smoother lines, with their tails positioned from bottom to top, correspond to $\xi=100$ to 300 .

the RW appearance is growing as the wave field evolves for both the disordered cases. This is substantially different from the IT although the system noise is considerably weak. Up to $\xi=300$, the probability $W$ roughly doubles from Case 0 to Case 1 , and increases by 5 times more from Case 1 to Case 2. For larger RWs of $h=12$, the situation is similar, as seen in Fig. 3(d), with the probability at $\xi=300$ increasing by about 6 times from Case 0 to Case 1, and 15 times more from Case 1 to Case 2.

Obviously, all these results in our numerical experiments support a "disorder-assisted" mechanism that leads to the enhancement of the RW occurrence. Nevertheless, how the disorder influences the content of the waves seems to be distinguishable as for Cases 1 and 2. We will analyze the linear and nonlinear spectra of the wave field in order to gain further knowledge.

The linear wave spectrum is

$$
S_{k}(\xi)=\left\langle\left|\psi_{k}(\xi)\right|^{2}\right\rangle,
$$

where $\psi_{k}(\xi)$ is the Fourier transformation of $\psi(\xi, \tau)$, defined as $\psi_{k}(\xi)=\frac{1}{L} \int_{-L / 2}^{L / 2} \psi(\xi, \tau) e^{-i k \tau} d \tau$, with the wave number $k=2 \pi n / L(n \in \mathbb{Z})$. For the MI-induced IT (Case 0), the initial spectrum consists of a central peak at $k=0$ corresponding to the plane wave and a small background due to the noisy perturbation. With evolution, the central peak spreads its energy into the sidebands. The modes within the instability band $k \in[-2,2]$ are amplified in the linear stage of MI, and from some time modes of the entire spectral band should grow due to the nonlinear interaction. When the stationary wave field is formed, the linear spectrum presents a fixed "onion dome" shape $[3,5]$, for which most of the nonlinear excitations are Akhmediev breathers.

Shown in Fig. 4 are the wave spectra measured at different $\xi$ for the disordered cases. We see that the shapes of the spectra are completely different from the asymptotic one of the IT. These relatively smooth spectra may correspond to the ensembles of solitons [5,48]. Widening of the spectra in evolution, with their tails being elevated, indicates enhanced interactions among those solitons. This widening accords with 

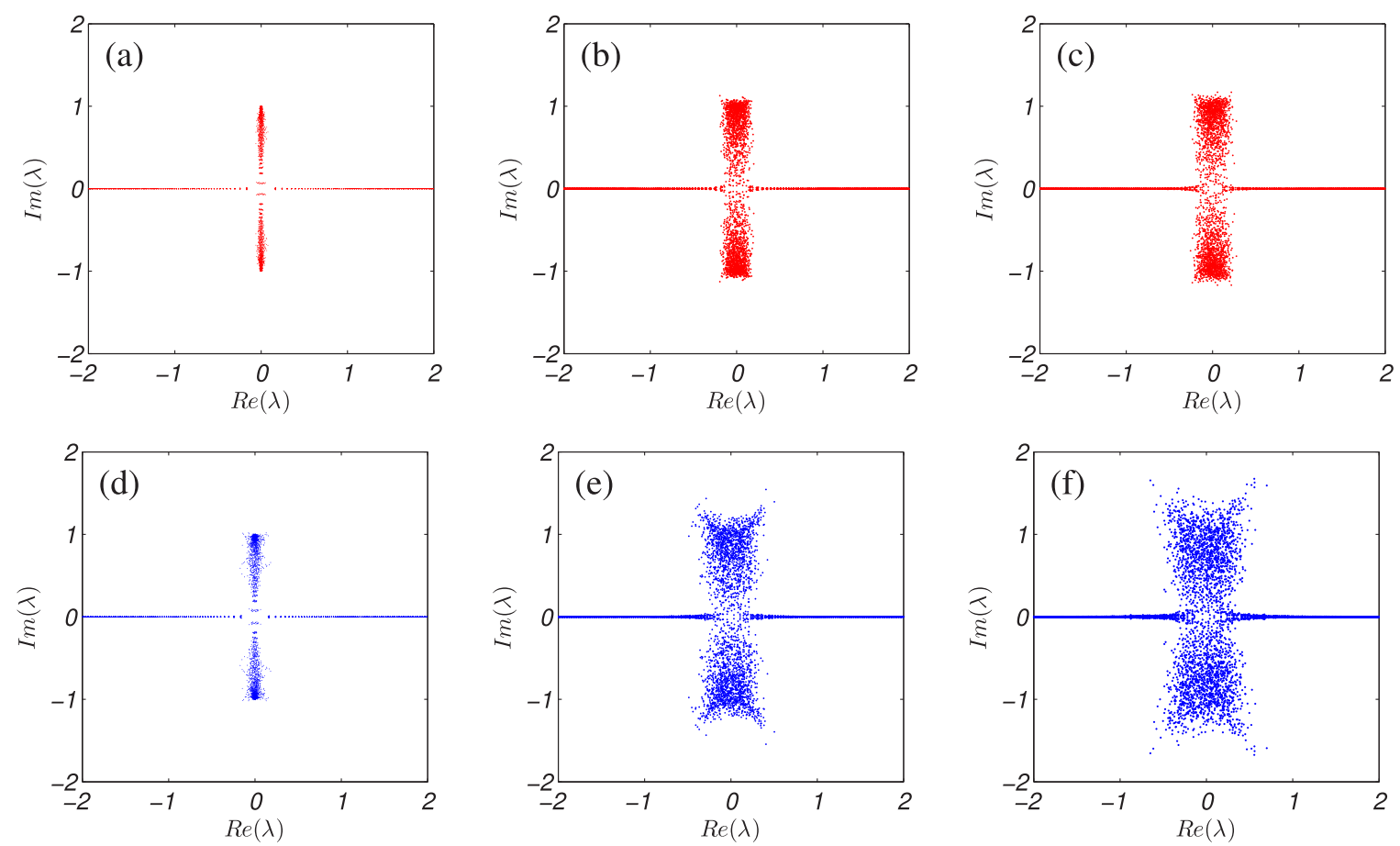

FIG. 5. Set of complex eigenvalues $\lambda$ of the NFT calculated for ten realizations of $\epsilon(\tau)$. Case 1 with the red plots: (a) $\xi=10$, (b) $\xi=100$, (c) $\xi=200$; and Case 2 with the blue plots: (d) $\xi=10$, (e) $\xi=100$, (f) $\xi=200$.

the increase of $M_{4}(\xi)$, which is a property of the integrable NLSE [43], and is still valid in our computation since the random perturbative components in Eq. (1) are small. Again, the variation of the spectrum with $\xi$ remains larger for Case 2 .

A deeper understanding of the wave content can be realized by using the tool of nonlinear Fourier transform (NFT), also known as the inverse scattering transform for integrable systems [49-51]. Like the conventional Fourier transform that decomposes the waveform into a set of spectral harmonics (e.g., see Fig. 4), the NFT decomposes waves into localized solitary eigenmodes and dispersive radiation components. This technique for the nonlinear spectrum was recently employed in IT to investigate ensembles of coherent structures such as breathers and solitons [4,5,48,52].

The basis of the NFT is to consider the Zakharov-Shabat spectral problem for a given wave function $\psi(\xi, \tau)$,

$$
\frac{d \mathbf{Y}}{d \tau}=\left(\begin{array}{cc}
-i \lambda & \psi \\
-\psi^{*} & i \lambda
\end{array}\right) \mathbf{Y}
$$

where $\mathbf{Y}(\xi, \tau ; \lambda)$ is a vector and $\lambda \in \mathbb{C}$ represents the spectral parameter. Finding the set of eigenvalues $\lambda$ by solving the linear equation (5) is of central importance for getting relevant information about the wave content. In principle, the NFT is rigorously applicable to the integrable NLSE with the spectrum of eigenvalues independent of $\xi$. However, it should be emphasized that our systems are nearly integrable [53], and the weak disordered perturbative effects produce some slow modulation of the spectral characteristics. Therefore we can still calculate the nonlinear spectrum using Eq. (5) at each point of $\xi$ and trace the evolution of these eigenvalues. Similar work was recently done to analyze nonintegrable
NLSEs with deterministic perturbations, e.g., with small dissipation [54-58].

Figure 5 shows the ensembles of eigenvalues collected at different $\xi$ for both Cases 1 and 2. The eigenvalues are numerically computed from Eq. (5) with periodic boundary conditions by using the Fourier collocation method [9,51] (also checked with the periodic Boffetta-Osborne method [51]). For the initial condition $\psi(0, \tau)=1$, the eigenvalues are purely imaginary, locating along the vertical axis, with the upper and lower limiting points $\lambda= \pm i$. They correspond to Akhmediev breathers whose amplitudes are less than 3. In Figs. 5(a) and 5(d), the eigenvalues begin to deviate from the imaginary axis but stay very close to it at short $\xi$. Thus the statistics of the wave field is close to that of IT. The eigenvalues appearing very near the real axis stand for small radiation waves. As $\xi$ evolves longer, the spectrum disperses randomly on the complex plane to form a specific global shape, as can be seen in the right four panels of Fig. 5. Most of the eigenvalues are apparently away from the imaginary axis, corresponding to solitons. Their amplitudes are twice the absolute values of the imaginary parts of the eigenvalues and their velocities are defined by the real parts of the eigenvalues.

Generally speaking, dispersion of the spectrum implies that many of the eigenvalues, but maybe not all of them, increase the absolute values of their real parts or imaginary parts (or both) in evolution. This means that the soliton velocities and soliton amplitudes become larger accordingly, resulting in enhanced soliton interactions and the increase of RW generation. This conclusion agrees with the tendency in Fig. 3 and also with the linear spectral analysis. However, the changes of the spectrum pattern for Cases 1 and 2 are visibly different. For Case 1, the spectrum becomes wider mainly along the real 
axis, suggesting that the RW enhancement is primarily due to the increase of the soliton velocities. For Case 2, distribution of the eigenvalues expands faster, along both the real and the imaginary axes. Thus, at the same point of $\xi$, the solitons acquire larger velocities and higher amplitudes than those for Case 1 , consequently producing stronger RWs. This provides us a qualitative explanation why the RW-related statistics is more remarkable for Case 2.

We want to emphasize that our results are fundamentally different from those by Soto-Crespo et al. [4], where their spectrum information has been given in the initial condition since for the integrable NLSE the eigenvalues are $\xi$ independent. Thus the RWs result from the elastic interaction of the high-amplitude solitons. In contrast, for Cases 1 and 2, the eigenvalue spectrum evolves with $\xi$. Some higher solitons are generated from inelastic interactions such as the fusion of solitons. These solitons may further raise the amplitudes of the RWs during their collisions. This is the reason that just weak noise is enough to apparently increase the probability of RWs for our cases.

\section{CONCLUSIONS AND OUTLOOKS}

To conclude, in this work we have provided numerical evidence of rogue wave enhancement in nearly integrable NLS systems with the presence of disorder. This enhancement is due to the dispersion of the nonlinear spectra of the wave field, which leads to the increase of the soliton interactions. We also have shown that random fluctuations of the nonlinearity would more significantly improve the probability of the occurrence of rogue waves. It is highly expected that our results will stimulate new relevant experimental work in fields such as in hydrodynamics [54,57] and optics [7,27,38,42] for understanding the interplay of disorder and nonlinearity. In addition, future work has to consider some interesting issues. An open question is the relationship between the Fourier (linear) spectrum and the NFT (nonlinear) spectrum. For small wave amplitudes, the NFT theory approaches the Fourier transform theory [50]. For larger waves (including solitons and rogue waves), these two spectra appear to show certain consistency in their dispersion (widening) speed and shape variation (see Figs. 4 and 5), which needs a detailed investigation. This may extend studies of the interactions between disorder and other perturbative effects such as the high-order nonlinearities [9] and dissipations [55,58]. On the other hand, considering evolving correlated disorder $\epsilon(\xi, \tau)$ gives rise to the concept "branched flow" for linear random waves $(g=0)$ [59-62]. Thus, incorporating enough large nonlinearity and creating stochastic solitons triggers, one may guess, some type of branchlike structures as "branched soliton flow." We hope these ideas yield many surprising results.

\section{ACKNOWLEDGMENTS}

We thank the referees for their careful and valuable comments. This work is supported by the National Natural Science Foundation of China (Grant No. 11902016), by the Fundamental Research Funds for the Central Universities, and by the Zhuoyue Talent Program of Beihang University.

\section{APPENDIX A: THE MODEL OF OPTICAL WAVES}

We briefly outline the connection of Eq. (1) with the optical-wave system. The evolution of spatial optical waves is described by the paraxial Schrödinger-type equation in $(1+1)$ dimensions [38,41,63],

$$
i \frac{\partial A}{\partial z}+\frac{1}{2 k_{0} n_{0}} \frac{\partial^{2} A}{\partial x^{2}}+k_{0} n_{L}(x) A+k_{0} n_{2}(x)|A|^{2} A=0
$$

where $A(x, z)$ is the slowly varying envelope of the optical field with the wave number $k_{0}=2 \pi / \lambda, n_{0}$ is the average linear refractive index and $n_{L}(x)$ is the linear fluctuation upon it, and $n_{2}(x)$ is the nonlinear Kerr coefficient. Such that the overall distribution of the refractive index stands as $n(x)=n_{0}+n_{L}(x)+n_{2}(x)|A|^{2}$. Equation (A1) has no temporal dependence, and the wave dynamics is only in space. The relevant optical system is uniform in the evolution direction (the axis $z$ ) but contains inhomogeneities (disorder) in the transverse direction (the axis $x$ ).

For Case 1, we consider the random fluctuations in the linear refractive index $\left[n_{2}(x) \equiv n_{20}\right]$. Introducing the dimensionless variables

$$
\xi=\frac{z}{k_{0} n_{0} x_{0}^{2}}, \quad \tau=\frac{x}{x_{0}}, \quad \psi(\xi, \tau)=k_{0} x_{0} \sqrt{n_{0} n_{20}} A
$$

where $x_{0}$ is the characteristic size of the optical beam, Eq. (A1) can be reduced into Eq. (1) of Case 1 with $\varepsilon(\tau)=$ $-k_{0}^{2} n_{0} x_{0}^{2} n_{L}(\tau)$. Employing the typical parameters $n_{0}=1.5$, $\lambda=0.6 \mu \mathrm{m}, x_{0}=5 \mu \mathrm{m}$, and $n_{L}<10^{-4}$, the requirement $|\varepsilon(\tau)|<0.4$ is completely fulfilled in our simulations.

For Case 2, the random inhomogeneities act on the nonlinear refractive index $\left[n_{L}(x) \equiv 0\right]$. We write $n_{2}(x)=n_{20}+$ $n_{2 L}(x)$, where $n_{20}$ is the average nonlinear index, superposed by the perturbation $n_{2 L}(x)$. Using also the scaling (A2), Eq. (1) of Case 2 can be obtained from Eq. (A1), with $g(\tau)=1+$ $n_{2 L}(\tau) / n_{20}$. In this work, $\left|n_{2 L}(\tau) / n_{20}\right|<0.02$ is kept.

\section{APPENDIX B: NUMERICAL CONFIGURATION AND ERRORS}

We here list some detailed parameters for our simulations: Equation (1) was integrated in the spatial box $\tau \in[-L / 2, L / 2], L=260 \pi$, with a uniform grid of $N=$ $2^{14}$ nodes (the grid size $\Delta \tau=L / N$ ); the evolution interval was $\xi \in[0,300]$ with the discretized step size $\Delta \xi=$ 0.0005; our Fourier spectral domain was consequently $k \in$ $[-\pi / \Delta \tau, \pi / \Delta \tau]$ with $\Delta k=2 \pi / L$.

The systems have two conserved integrals over the evolution coordinate, the mass $S(\xi)$, and Hamiltonian $H(\xi)$,

$$
\begin{gathered}
S=\frac{1}{L} \int|\psi|^{2} d \tau \\
H=\frac{1}{L} \int\left[\frac{1}{2}\left|\frac{\partial \psi}{\partial \tau}\right|^{2}+\frac{1}{2} g(\tau)|\psi|^{4}+\varepsilon(\tau)|\psi|^{2}\right] d \tau .
\end{gathered}
$$

We checked the preservation of these integrals by tracking their relative errors as $S_{r}(\xi)=|S(\xi)-S(0)| /|S(0)|$ and $H_{r}(\xi)=|H(\xi)-H(0)| /|H(0)|$. A typical result is presented in Fig. 6, where we see that $S_{r}$ and $H_{r}$ are considerably small for both cases. For all realizations (others not shown for brevity), the errors are generally bounded at the level of 

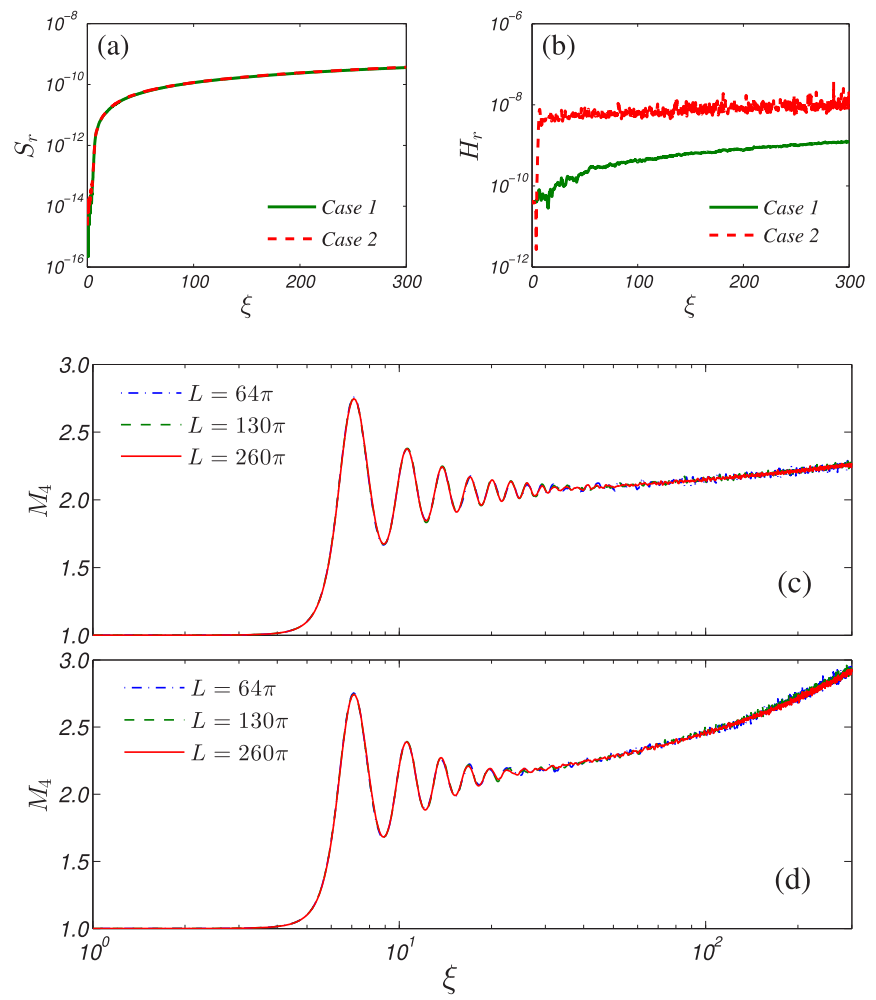

FIG. 6. Evolution of (a) the relative mass error $S_{r}$ and (b) the relative Hamiltonian error $H_{r}$ for a specific realization of randomness. Plots of $M_{4}(\xi)$ for different sizes of the integration box: $L=64 \pi$ (blue dashed-dotted line), $L=130 \pi$ (green dashed line), and $L=$ $260 \pi$ (red solid line). Here (c) is for Case 1 while (d) is for Case 2 (the results are averaged over the ensembles of 100 realizations of randomness). Note that the lines in (a), (c) and (d) are almost overlapped.

$S_{r}<10^{-9}$ and $H_{r}<10^{-7}$. Thus the mass and Hamiltonian are well conserved in our simulations.

On the other hand, our numerical scheme uses the periodic boundary conditions, for which the waves touching one boundary may be "reflected" back to the wave field from the other boundary (this computational effect can be generally found, e.g., in $[64,65])$. In principle, the results tend to the case of a turbulent wave field without boundaries when the box size $L$ becomes larger. We have tested various $L$ to make sure that our results do not change with the increase of $L$, as seen in Figs. 6(c) and 6(d). Thus $L=260 \pi$ used is large enough to keep those results free of numerical artifacts.

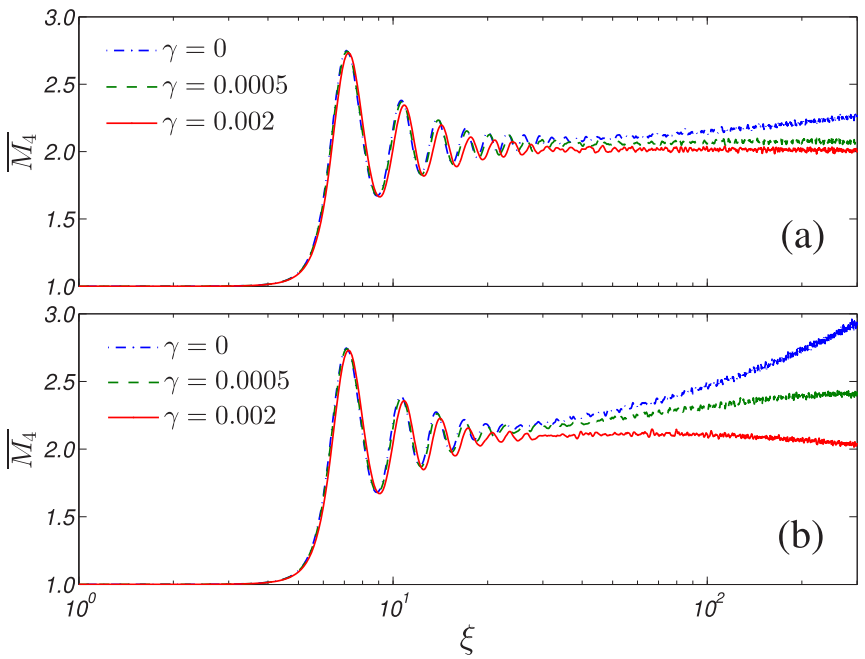

FIG. 7. Evolution of the normalized fourth-order moment $\overline{M_{4}}(\xi)$ for different loss parameters: $\gamma=0$ (upper blue dashed-dotted line), $\gamma=0.0005$ (middle green dashed line), and $\gamma=0.002$ (lower red solid line). Here (a) is for Case 1 and (b) is for Case 2 (the results are averaged over the ensembles of 100 realizations of randomness).

\section{APPENDIX C: EFFECT OF LINEAR LOSS}

We briefly consider the effect of linear loss in the systems. The loss term $R=-i \gamma \psi$ is incorporated into the right-hand side of Eq. (1), where $\gamma$ is the normalized loss per propagation distance. For this situation, the mass of the wave field is dissipated as $S(\xi)=S(0) \exp (-2 \gamma \xi)$, and the fourth-order moment should be normalized: $\overline{M_{4}}(\xi)=M_{4}(\xi) / S^{2}(\xi)$. Two typical examples are presented in Fig. 7 where the loss is small: For $\gamma=0.0005$ about $74 \%$ of the initial mass is preserved at $\xi=300$, while for $\gamma=0.002$ this ratio is $30 \%$.

With a loss, the value of $\overline{M_{4}}$ is generally smaller than that of the lossless case. However, when $\xi \gtrsim 20, \overline{M_{4}}$ increases with $\xi$ for a certain interval, then begins to decrease for the longer propagation distance. This two-phase effect holds for both Cases 1 and 2, which is due to the competition between the disorder and the linear loss. When the mass dissipation is small, the disorder still can improve the soliton interactions effectively; if more mass is dissipated, the wave amplitudes become smaller, and $\overline{M_{4}}$ may finally approach the value of 2 . Thus the linear loss reduces the probability of rogue wave occurrence, especially for a long propagation distance.
[1] V. E. Zakharov, Turbulence in integrable systems, Stud. Appl. Math. 122, 219 (2009).

[2] E. Pelinovsky and A. Sergeeva, Numerical modeling of the KdV random wave field, Eur. J. Mech. B Fluids 25, 425 (2006).

[3] D. S. Agafontsev and V. E. Zakharov, Integrable turbulence and formation of rogue waves, Nonlinearity 28, 2791 (2015).

[4] J. M. Soto-Crespo, N. Devine, and N. Akhmediev, Integrable Turbulence and Rogue Waves: Breathers or Solitons? Phys. Rev. Lett. 116, 103901 (2016).
[5] N. Akhmediev, J. M. Soto-Crespo, and N. Devine, Breather turbulence versus soliton turbulence: Rogue waves, probability density functions, and spectral features, Phys. Rev. E 94, 022212 (2016).

[6] P. Walczak, S. Randoux, and P. Suret, Optical Rogue Waves in Integrable Turbulence, Phys. Rev. Lett. 114, 143903 (2015).

[7] P. Suret, R. E. Koussaifi, A. Tikan, C. Evain, S. Randoux, C. Szwaj, and S. Bielawski, Single-shot observation of optical rogue waves in integrable turbulence using time microscopy, Nat. Commun. 7, 13136 (2016). 
[8] I. Redor, E. Barthélemy, H. Michallet, M. Onorato, and N. Mordant, Experimental Evidence of a Hydrodynamic Soliton Gas, Phys. Rev. Lett. 122, 214502 (2019).

[9] J. Yang, Nonlinear Waves in Integrable and Nonintegrable Systems (SIAM, Philadelphia, 2010).

[10] F. Copie, S. Randoux, and P. Suret, The Physics of the onedimensional nonlinear Schrödinger equation in fiber optics: Rogue waves, modulation instability and self-focusing phenomena, Rev. Phys. 5, 100037 (2020).

[11] V. E. Zakharov and A. A. Gelash, Nonlinear Stage of Modulation Instability, Phys. Rev. Lett. 111, 054101 (2013).

[12] M. Närhi, B. Wetzel, C. Billet, S. Toenger, T. Sylvestre, J.M. Merolla, R. Morandotti, F. Dias, G. Genty, and J. M. Dudley, Real-time measurements of spontaneous breathers and rogue wave events in optical fibre modulation instability, Nat. Commun. 7, 13675 (2016).

[13] A. E. Kraych, D. Agafontsev, S. Randoux, and P. Suret, Statistical Properties of the Nonlinear Stage of Modulation Instability in Fiber Optics, Phys. Rev. Lett. 123, 093902 (2019).

[14] Z. Rapti, P. G. Kevrekidis, A. Smerzi, and A. R. Bishop, Variational approach to the modulational instability, Phys. Rev. E 69, 017601 (2004).

[15] N. Akhmediev, A. Ankiewicz, and M. Taki, Waves that appear from nowhere and disappear without a trace, Phys. Lett. A 373, 675 (2009).

[16] N. Akhmediev, J. M. Soto-Crespo, and A. Ankiewicz, Extreme waves that appear from nowhere: On the nature of rogue waves, Phys. Lett. A 373, 2137 (2009).

[17] S. Toenger, T. Godin, C. Billet, F. Dias, M. Erkintalo, G. Genty, and J. M. Dudley, Emergent rogue wave structures and statistics in spontaneous modulation instability, Sci. Rep. 5, 10380 (2015).

[18] M. Onorato, A. R. Osborne, M. Serio, and S. Bertone, Freak Waves in Random Oceanic Sea States, Phys. Rev. Lett. 86, 5831 (2001).

[19] C. Kharif and E. Pelinovsky, Physical mechanisms of the rogue wave phenomenon, Eur. J. Mech. B Fluids 22, 603 (2003).

[20] K. Dysthe, H. E. Krogstad, and P. Müller, Oceanic Rogue Waves, Annu. Rev. Fluid Mech. 40, 287 (2008).

[21] M. Onorato, S. Residori, U. Bortolozzo, A. Montinad, and F. T. Arecchi, Rogue waves and their generating mechanisms in different physical contexts, Phys. Rep. 528, 47 (2013).

[22] A. Armaroli, C. Conti, and F. Biancalana, Rogue solitons in optical fibers: a dynamical process in a complex energy landscape? Optica 2, 497 (2015).

[23] A. Chabchoub, N. Hoffmann, M. Onorato, and N. Akhmediev, Super Rogue Waves: Observation of a Higher-Order Breather in Water Waves, Phys. Rev. X 2, 011015 (2012).

[24] B. Frisquet, B. Kibler, and G. Millot, Collision of Akhmediev Breathers in Nonlinear Fiber Optics, Phys. Rev. X 3, 041032 (2013).

[25] E. G. Charalampidis, J. Lee, P. G. Kevrekidis, and C. Chong, Phononic rogue waves, Phys. Rev. E 98, 032903 (2018).

[26] S. Perrone, R. Vilaseca, J. Zamora-Munt, and C. Masoller, Controlling the likelihood of rogue waves in an optically injected semiconductor laser via direct current modulation, Phys. Rev. A 89, 033804 (2014).

[27] J. M. Dudley, F. Dias, M. Erkintalo, and G. Genty, Instabilities, breathers and rogue waves in optics, Nat. Photonics 8, 755 (2014).
[28] T. I. Lakoba, Effect of noise on extreme events probability in a one-dimensional nonlinear Schrödinger equation, Phys. Lett. A 379, 1821 (2015).

[29] A. S. Pikovsky and D. L. Shepelyansky, Destruction of Anderson Localization by Weak Nonlinearity, Phys. Rev. Lett. 100, 094101 (2008).

[30] S. Flach, D. O. Krimer, and C. Skokos, Universal Spreading of Wave Packets in Disordered Nonlinear Systems, Phys. Rev. Lett. 102, 024101 (2009).

[31] I. Vakulchyk, M. V. Fistul, and S. Flach, Wave Packet Spreading with Disordered Nonlinear Discrete-Time Quantum Walks, Phys. Rev. Lett. 122, 040501 (2019).

[32] S. Nazarenko, A. Soffer, and M.-B. Tran, On the wave turbulence theory for the nonlinear Schrödinger equation with random potentials, Entropy 21, 823 (2019).

[33] S. Fishman, Y. Krivolapov, and A. Soffer, The nonlinear Schrödinger equation with a random potential: Results and puzzles, Nonlinearity 25, R53 (2012).

[34] Z.-Y. Sun, S. Fishman, and A. Soffer, Soliton mobility in disordered lattices, Phys. Rev. E 92, 040903(R) (2015).

[35] Z.-Y. Sun and X. Yu, Transport of nonautonomous solitons in two-dimensional disordered media, Ann. Phys. (Berlin) 529, 1600323 (2017).

[36] Z.-Y. Sun and X. Yu, Anomalous diffusion of discrete solitons driven by evolving disorder, Phys. Rev. E 101, 062211 (2020).

[37] C. Conti, Complex light: Dynamic phase transitions of a light beam in a nonlinear nonlocal disordered medium, Phys. Rev. E 72, 066620 (2005).

[38] T. Schwartz, G. Bartal, S. Fishman, and M. Segev, Transport and Anderson localization in disordered two-dimensional photonic lattices, Nature (London) 446, 52 (2007).

[39] J. E. Lye, L. Fallani, M. Modugno, D. S. Wiersma, C. Fort, and M. Inguscio, Bose-Einstein Condensate in a Random Potential, Phys. Rev. Lett. 95, 070401 (2005).

[40] T. Schulte, S. Drenkelforth, J. Kruse, W. Ertmer, J. Arlt, K. Sacha, J. Zakrzewski, and M. Lewenstein, Routes Towards Anderson-Like Localization of Bose-Einstein Condensates in Disordered Optical Lattices, Phys. Rev. Lett. 95, 170411 (2005).

[41] F. Abdullaev and J. Garnier, Optical solitons in random media, Prog. Opt. 48, 35 (2005).

[42] Y. Sharabi, H. H. Sheinfux, Y. Sagi, G. Eisenstein, and M. Segev, Self-Induced Diffusion in Disordered Nonlinear Photonic Media, Phys. Rev. Lett. 121, 233901 (2018).

[43] M. Onorato, D. Proment, G. El, S. Randoux, and P. Suret, On the origin of heavy-tail statistics in equations of the Nonlinear Schrödinger type, Phys. Lett. A 380, 3173 (2016).

[44] R. Jordan and C. Josserand, Self-organization in nonlinear wave turbulence, Phys. Rev. E 61, 1527 (2000).

[45] A. I. D'yachenko, V. E. Zakharov, A. N. Pushkarev, V. F. Shvets, and V. V. Yan'kov, Soliton turbulence in nonintegrable wave systems, Sov. Phys. JETP 69, 1144 (1989).

[46] B. Rumpf and A. C. Newell, Localization and coherence in nonintegrable systems, Physica D 184, 162 (2003).

[47] A. Picozzi and J. Garnier, Incoherent Soliton Turbulence in Nonlocal Nonlinear Media, Phys. Rev. Lett. 107, 233901 (2011).

[48] A. A. Gelash and D. S. Agafontsev, Strongly interacting soliton gas and formation of rogue waves, Phys. Rev. E 98, 042210 (2018). 
[49] M. J. Ablowitz and H. Segur, Solitons and the Inverse Scattering Transform (SIAM, Philadelphia, 1981).

[50] A. R. Osborne, Nonlinear Ocean Waves and the Inverse Scattering Transform (Academic, San Diego, 2010).

[51] S. K. Turitsyn, J. E. Prilepsky, S. T. Le, S. Wahls, L. L. Frumin, M. Kamalian, and S. A. Derevyanko, Nonlinear Fourier transform for optical data processing and transmission: advances and perspectives, Optica 4, 307 (2017).

[52] A. Gelash, D. Agafontsev, V. Zakharov, G. El, S. Randoux, and P. Suret, Bound State Soliton Gas Dynamics Underlying the Spontaneous Modulational Instability, Phys. Rev. Lett. 123, 234102 (2019).

[53] Y. S. Kivshar and B. A. Malomed, Dynamics of solitons in nearly integrable systems, Rev. Mod. Phys. 61, 763 (1989).

[54] S. Randoux, P. Suret, A. Chabchoub, B. Kibler, and G. El, Nonlinear spectral analysis of Peregrine solitons observed in optics and in hydrodynamic experiments, Phys. Rev. E 98, 022219 (2018).

[55] I. S. Chekhovskoy, O. V. Shtyrina, M. P. Fedoruk, S. B. Medvedev, and S. K. Turitsyn, Nonlinear Fourier Transform for Analysis of Coherent Structures in Dissipative Systems, Phys. Rev. Lett. 122, 153901 (2019).

[56] S. Sugavanam, M. K. Kopae, J. Peng, J. E. Prilepsky, and S. K. Turitsyn, Analysis of laser radiation using the Nonlinear Fourier transform, Nat. Commun. 10, 5663 (2019).
[57] F. Bonnefoy, A. Tikan, F. Copie, P. Suret, G. Ducrozet, G. Prabhudesai, G. Michel, A. Cazaubiel, E. Falcon, G. El, and S. Randoux, From modulational instability to focusing dam breaks in water waves, Phys. Rev. Fluids 5, 034802 (2020).

[58] S. K. Turitsyn, I. S. Chekhovskoy, and M. P. Fedoruk, Nonlinear Fourier transform for characterization of the coherent structures in optical microresonators, Opt. Lett. 45, 3059 (2020).

[59] E. J. Heller, R. Fleischmann, and T. Kramer, Branched flow, arXiv:1910.07086.

[60] A. Patsyk, U. Sivan, M. Segev, and M. A. Bandres, Observation of branched flow of light, Nature (London) 583, 60 (2020).

[61] G. Green and R. Fleischmann, Branched flow and caustics in nonlinear waves, New. J. Phys. 21, 083020 (2019).

[62] M. Mattheakis and G. P. Tsironis, Extreme Waves and branched flows in optical media, Quodons in Mica, Springer Series in Materials Science Vol. 221 (Springer International, Cham, 2015), pp. 425-454.

[63] C. P. Jisha, A. Alberucci, R.-K. Lee, and G. Assanto, Deflection and trapping of spatial solitons in linear photonic potentials, Opt. Express 21, 18646 (2013).

[64] K. Tai, A. Hasegawa, and N. Bekki, Fission of optical solitons induced by stimulated Raman effect, Opt. Lett. 13, 392 (1988).

[65] A. Hasegawa and M. Matsumoto, Optical Solitons in Fibers (Springer-Verlag, Berlin, 2003). 